\title{
O Significado dos Anabolizantes para os Adolescentes
}

\author{
Monique Santos Carregosa ${ }^{1}$ \\ Universidade Federal de Sergipe, São Cristóvão, SE, Brasil \\ André Faro \\ Departamento de Psicologia da Universidade Federal de Sergipe, São Cristóvão, SE, Brasil
}

\section{Resumo}

Este estudo objetivou conhecer o conceito de anabolizantes e identificar benefícios e malefícios do uso, segundo adolescentes. Participaram 508 estudantes do ensino médio de duas escolas públicas, com 16,2 anos, em média. Utilizou-se um questionário semiaberto abordando o conceito, os benefícios e malefícios dos anabolizantes. O corpus foi único e as respostas foram analisadas com o software IRAMUTEQ, através de Classificação Hierárquica Descendente (CHD). Os resultados indicaram três principais classes: (1) Conceitos de anabolizantes, (2) Benefícios derivados dos anabolizantes, e (3) Prejuízos derivados dos anabolizantes. A primeira apontou três principais conceitos de anabolizantes: (a) "algo que aumenta a massa muscular"; (b) "melhora o desempenho na academia"; e (c) "paradoxo: mau e bom". A classe dos benefícios demonstrou que os adolescentes não destacam apenas os ganhos, mas também os custos, apesar das vantagens serem sobrepostas. A terceira classe indicou que há certo conhecimento a respeito dos malefícios dos anabolizantes, mas que ainda pode representar riscos e levar a uma subestimação dos efeitos colaterais decorrentes do uso. Portanto, os adolescentes se remetem à preocupação de seu grupo quanto à aparência física e apresentam um discurso sobre os anabolizantes voltado para a saúde, seja através de uma falsa aparência saudável ou dos prejuízos.

Palavras-chave: Anabolizantes, significado, adolescentes, corpo.

\section{The Meaning of the Steroids Anabolics for Teenagers}

\begin{abstract}
This study investigated the concept of anabolic steroids and identified benefits and harms of using according to adolescents. There were 508 high school students from two public schools, with 16.2 years on average. We used a semi-open questionnaire addressing the concept, the benefits and harms derived from steroids. The corpus was unique and the answers were analyzed with the software IRAMUTEQ, by Hierarchical Classification Descending (CHD). The results indicated three main classes: (1) Concepts of steroids, (2) Benefits derived from steroids, and (3) Losses derived from steroids. The first indicated three main concepts of steroids: (a) "something that increases muscle mass"; (b) "improves performance at the gym"; and (c) "paradox: good and bad." The class of benefits showed that adolescents do not highlight only gains, but also the costs, despite the advantages being overlapping. The third class indicated that there is some knowledge about the harms of anabolic steroids, but may create hazards and lead to an underestimation of the side effects of using. Therefore, adolescents referred the concern of his group regarding physical appearance and they present a discourse about steroids focused on health, either through a false healthy appearance or emphasize the damages.
\end{abstract}

Keywords: Steroids anabolics, meaning, teenagers, body.

Endereço para correspondência: Universidade Federal de Sergipe, Programa de Pós-Graduação em Psicologia Social, Av. Marechal Rondon, s/n, Conjunto Rosa Elze, São Cristóvão, SE, Brasil 49000-000. Fone: 79 88316865. E-mail: monyky09@hotmail.com e andre.faro.ufs@gmail.com 


\section{El Significado de los Esteroides Anabólicos para Adolescentes}

\section{Resumen}

Este estudio investigó el concepto de esteroides anabólicos e identificó riesgos y beneficios de su utilización, según adolescentes. Participarón 508 estudiantes secundaristas de dos escuelas públicas, con 16,2 años de media. Se utilizó un cuestionario semi-abierto abordando el concepto, beneficios y daños de los esteroides. Fue un solo corpus y se analizaron las respuestas con el software IRAMUTEQ, mediante Clasificación Jerárquica Descendente (CJD). Los resultados indicaron tres clases: (1) Conceptos de los esteroides, (2) Los beneficios derivados de los esteroides, y (3) Pierdas relacionadas a los esteroides. En la primera clase fueron identificados tres conceptos: (a) "algo que aumenta la masa muscular"; (b) "mejora el rendimiento en el gimnasio"; y (c) "paradoja: bien y mal." La clase de beneficios demostró que los adolescentes no destacan sólo los beneficios, sino también los costes, a pesar de las ventajas que se superponen. La tercera clase indicó que hay poco conocimiento acerca de los daños causados por los anabólicos, pero también pueden representar riesgos y subestimar sus perjuicios. Por lo tanto, los adolescentes remiten la preocupación de su grupo con la imagen física y presentan un discurso sobre la salud, sea a través de una falsa apariencia saludable o relacionado a los daños.

Palabras clave: Esteroides anabólicos, concepto, adolescentes, cuerpo.

A pesquisa realizada pelo Centro Brasileiro de Informações sobre Drogas Psicotrópicas (CEBRID, 2010) mostrou que $1,4 \%$ dos estudantes brasileiros já colocou a saúde em risco com o uso de anabolizantes. Os principais motivos se referiram ao crescente uso para fins estéticos sem prescrição médica, aquisição ilícita e motivada, principalmente, em função do baixo custo, fácil acesso e resposta em curto prazo (Iriart, Chaves, \& Orleans, 2009; Oliveira, 2012). Diante desse quadro, o problema do uso indevido de anabolizantes se torna mais abrangente, na medida em que sua popularização pode significar a extensão do consumo entre os jovens pertencentes a diferentes classes sociais.

Os esteroides anabólico-androgênicos (EAA), popularmente chamados de anabolizantes ou "bombas", são substâncias sintéticas à base de testosterona (Darke, Torok, \& Duflou, 2014), procuradas tanto pela capacidade de aumentar a massa muscular - efeitos anabólicos - quanto pelo desenvolvimento de características sexuais masculinas - efeitos androgênicos -, sendo a testosterona e a oxandrolona algumas das principais substâncias utilizadas (Pope \& Kanayama, 2012).

A aplicação de anabolizantes é clinicamente recomendável para o tratamento de doenças como deficiência de andrógenos, câncer de mama, estimulação do desenvolvimento ósseo, dos músculos, do apetite e da puberdade, interrupção prematura do surto de crescimento na adolescência, dentre outras patologias (Amsterdam, Opperhuizen, \& Hartgens, 2010; F. L. Rocha, Roque, \& Oliveira, 2007). Quando utilizados por recomendação médica, normalmente os efeitos clínicos condizem com o esperado, mas, ainda assim, há o risco de efeitos colaterais, ou até mesmo os decorrentes do uso inadequado. Contudo, percebe-se um uso recreativo e abusivo especialmente em atletas e fisiculturistas, a fim de melhorar o desempenho físico (A. G. Machado \& Ribeiro, 2004) e principalmente por usuários do sexo masculino com idade predominante entre o final da adolescência e trinta e poucos anos (Darke et al., 2014).

Estudos a respeito dos efeitos prejudiciais dos anabolizantes evidenciam que eles podem ocasionar problemas que vão desde os sintomas mais simples, como dores de cabeça, diarreias, acnes e dor no local da injeção até os mais complexos e às vezes irreversíveis, tais como: problemas no Sistema Nervoso (Pedroso, 2012), cardíacos (F. L. Rocha et al., 2007), hepáticos (Socas et al., 2005), infertilidade masculina (Nangia, 2014) e morbidade hospitalar (S. H. A. Silva, 2013). Há ainda os efeitos psicológicos e neuropsiquiátricos que, apesar de não se- 
rem muito divulgados, impactam negativamente sobre a saúde e ajustamento do indivíduo, visto que os anabolizantes podem induzir efeitos secundários psiquiátricos (Pagonis, Angelopoulos, Koukoulis, \& Hadjichristodoulou, 2005) e possuem relação tanto com uma vida antissocial e com o transtorno de conduta (Thiblin \& Pärlklo, 2002), quanto com a manifestação de comportamentos de risco, em especial nos adolescentes (Martins et al., 2005).

No público adolescente, investigações recentes apontam para um assíduo e crescente aumento do consumo de anabolizantes, cuja finalidade não é competitiva, mas sim atingir um corpo "belo" e considerado "ideal". Essa visão tem sido cada vez mais influenciada pela supervalorização dos corpos em sociedades de consumo pós-industriais e do imediatismo por elas propagado (Oliveira, 2012). Daí a preocupação com os adolescentes, que podem tentar encurtar esse caminho recorrendo ao uso de esteroides anabolizantes.

Em meio a uma possível insatisfação com o corpo, necessidade de elevação da autoestima e até distorção da imagem corporal, os esteroides podem surgir como a "solução imediata" para o ganho muscular almejado (M. Rocha, Mota, \& Matos, 2011). Iriart et al. (2009) descrevem que esses fatores são reforçados pelo padrão "ideal" disseminado pela mídia e pelo receio que os usuários tem de serem desvalorizados ou excluídos do grupo, o que favorece ainda mais o uso de anabolizantes.

$\mathrm{Na}$ adolescência, os indivíduos passam por modificações que podem gerar um desejo de autoafirmação e a expectativa de bela imagem corporal, bem como sofrem pressões ligadas a estereótipos de gênero, as quais ditam que, enquanto a força muscular nos meninos significa beleza e masculinidade, por outro lado um corpo com curvas bem definidas tornam as meninas mais sedutoras e sensuais (Ozella, 2002). Conforme Almeida, Rodrigues e Simões (2007), como a imagem corporal ainda está em processo de construção, podem surgir conflitos entre a imagem fantasiada e a percepção do próprio corpo, o que pode levar os adolescentes a comportamentos de risco à saúde, a exemplo do uso indevido de anabolizantes, por ignorarem diferenças individuais de maturação.

Alguns estudos alertam quanto ao aumento do uso de anabolizantes entre adolescentes (Alan, 2009; CEBRID, 2010; Sociedade Brasileira de Endocrinologia e Metabologia, 2014), o que enfatiza a necessidade de ampliar o conhecimento sobre a temática, em virtude de sua relevância para a saúde pública e, ao mesmo tempo, para o contexto escolar, por ser um problema que demanda também uma intervenção educacional. Além disso, esses trabalhos reforçam a opinião de que a falta de informação também se estende para a literatura científica, pois no Brasil o consumo para fins estéticos dos anabolizantes ainda é pouco estudado (Iriart et al., 2009) e a incidência e prevalência do seu uso ilícito entre adolescentes também carecem de pesquisas (Araújo, 2003; Manetta \& Silveira, 2000; Ribeiro, 2001).

Pesquisadores como Iriart e Andrade (2002), Iriart et al. (2009), Oliveira (2012) e Santos, Mendonça, Santos, Silva e Tavares (2006) têm se preocupado com a problemática dos anabolizantes, mais especificamente com as motivações e a percepção dos usuários acerca dos esteroides. Por essa via, analisar os conceitos formulados por determinados indivíduos acerca de um fenômeno ou objeto se mostra importante para o estudo dos significados compartilhados em grupos sociais, pois essas formulações possuem um papel comunicacional entre seus membros. Tais conceitos atuam sobre as qualidades das trocas sociais estabelecidas pelos indivíduos, modelando influências mútuas e negociações interpessoais implícitas, o que favorece a criação de um repertório comum de explicações, regras e comportamentos aplicáveis em seu cotidiano (L. M. G. S. Machado, 2010; Santos et al., 2006).

No caso dos adolescentes, sabe-se ainda suas crenças figuram como um importante preditor de comportamentos de saúde (Antunes et al., 2006; Simões, 2010) e, por isso, compreender como eles percebem os esteroides viabiliza a análise de como os mesmos avaliam e se posicionam diante dessa problemática. Dessa forma, o presente estudo visa a conhecer os 
principais significados atribuídos por adolescentes aos anabolizantes, bem como identificar os benefícios e malefícios decorrentes do uso dessas substâncias, segundo o discurso dos participantes.

\section{Método}

\section{Participantes}

Participaram 508 adolescentes, de ambos os sexos, sendo a maioria do sexo feminino $(59,3 \%)$, com idade média de 16,2 anos [Desvio-Padrão $(D P)=1,11$; Mínimo $=14$ - Máximo $=19]$. Todos eram estudantes do ensino médio (turmas de $1^{\circ}, 2^{\circ}$ e $3^{\circ}$ ano), dos turnos matutino e vespertino, de uma escola da rede pública da capital $(n=252)$ e uma do interior de Sergipe ( $n$ $=256$ ). Destes, somente $27,4 \%$ eram praticantes de musculação. No momento da coleta, houve apenas sete recusas. Outros 19 adolescentes não preencheram alguma resposta a respeito dos anabolizantes, dentre as constantes nos instrumentos de pesquisa, tendo sido excluídos os seus questionários.

\section{Instrumentos e Procedimentos}

O estudo foi aprovado pelo Comitê de Ética em Pesquisa com Seres Humanos da Universidade Federal de Sergipe - UFS (CAAE 35505314.0.0000.5546). Mediante a autorização das escolas, as quais foram escolhidas por conveniência, os questionários foram aplicados durante a semana, utilizando-se de 15 minutos de uma aula, conforme consentimento do professor que estava em sala e assinatura do Termo de Consentimento Livre e Esclarecido pelos participantes.

Utilizou-se um questionário com 3 perguntas semi-abertas: "para você, o que é o anabolizante"?; "quais os seus benefícios (consequências positivas)"? e "quais os prejuízos (consequências negativas) que os anabolizantes podem trazer a quem usa?

\section{Análise dos Dados}

As respostas das 3 perguntas foram analisadas pelo IRAMUTEQ (Interface de $R$ pour les Analyses Multidimensionnel- les de Textes et de Questionnaires), um software que permite fazer análises estatísticas sobre corpus textuais. O corpus de análise foi único, constituído pelas respostas dos participantes às 3 questões semiabertas, as quais foram categorizadas em três classes: conceitos, benefícios e malefícios. Dentre as análises possíveis no IRAMUTEQ utilizou-se a Classificação Hierárquica Descendente (CHD; Camargo \& Justo, 2013).

O método da CHD permite obter classes de segmentos de texto que apresentam vocabulário semelhante entre si e/ou vocabulário diferente das demais classes, e também calcula a distância e a proximidade de suas relações com testes do Qui-Quadrado. Com efeito, o IRAMUTEQ classifica os léxicos num dendograma, agregando-os de acordo com a frequência relativa e o valor de Qui-Quadrado do léxico correspondente na classe (Camargo, 2005).

Por meio do dendograma, extraiu-se o conjunto de léxicos ou palavras reunidas nas classes temáticas e identificaram-se as de maior relação de importância entre classe e palavra, representadas pelos valores de Qui-quadrado. A partir da análise dos principais léxicos, a pesquisadora construiu um discurso simbólico para representar cada classe.

\section{Resultados e Discussão}

O corpus analisado obteve 508 UCI (questionários), 16.882 ocorrências (número de palavras), e frequência de 4,9 palavras por resposta. O dendograma foi formado por 3 classes temáticas conforme as semelhanças entre os segmentos de texto, a saber: (a) Conceitos de anabolizantes, (b) Benefícios derivados dos anabolizantes, e (c) Prejuízos derivados dos anabolizantes (Figura 1).

A seguir, será realizada a análise por classe, conforme os conteúdos a partir da organização do dendograma. 


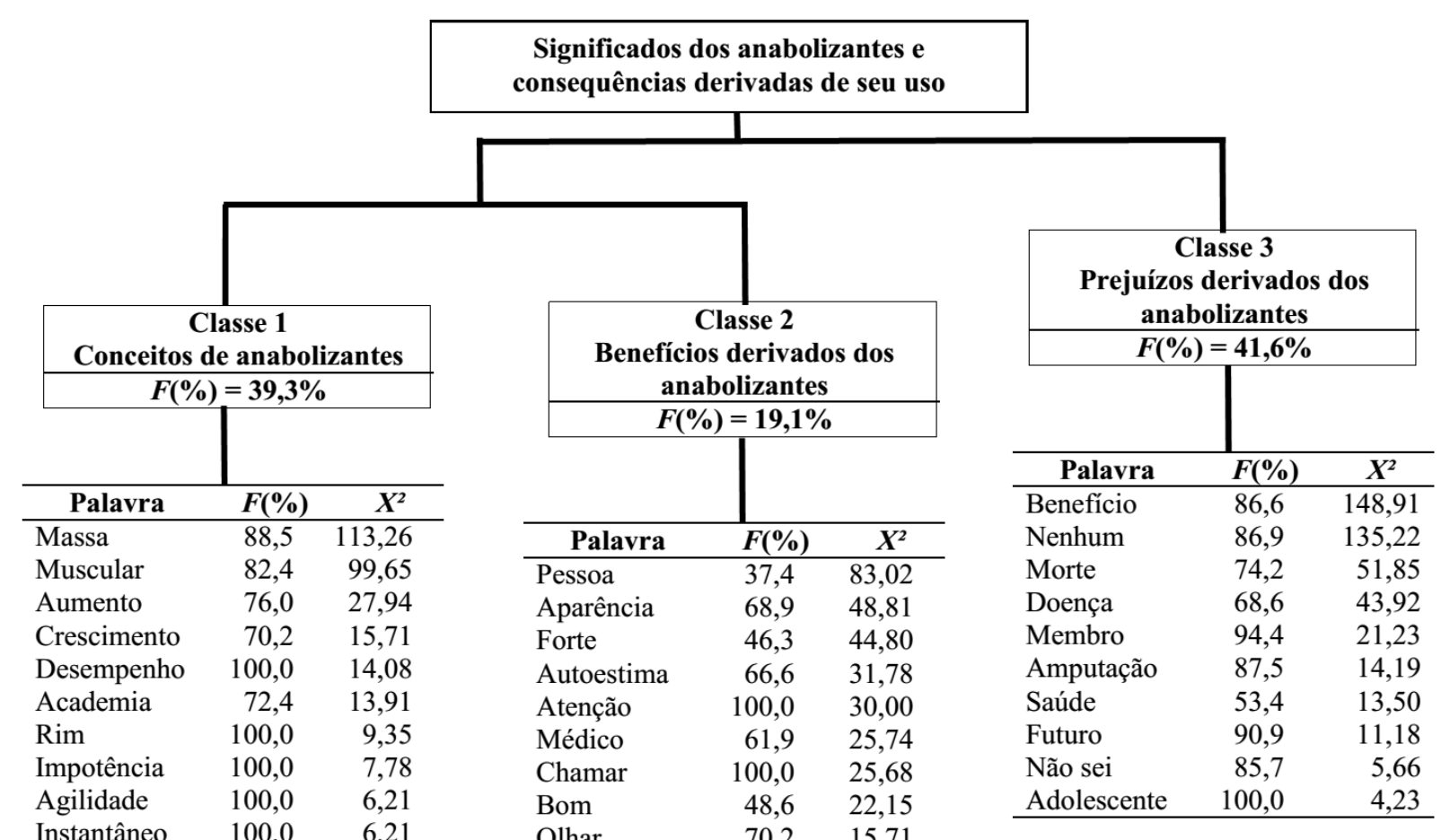

Figura 1. Dendograma e classes léxicas dos significados dos anabolizantes e consequências derivadas do uso.

\section{Classe 1: Conceitos de Anabolizantes $(39,3 \%)$}

Esta classe descreve os conceitos que os adolescentes formulam sobre os anabolizantes, sendo constatadas três principais definições, extraídas a partir da análise contextual dos principais termos (maior qui-quadrado) da classe no dendograma. A primeira foi formada com as quatro primeiras palavras evocadas pelos sujeitos, compreendendo desde o termo massa $(88,5 \%)$ até a palavra crescimento $(70,2 \%)$, trazendo a ideia de "algo que aumenta a massa muscular". A segunda definição encontrada foi "melhora o desempenho na academia", sendo que todos os segmentos do texto da amostra obtiveram o termo desempenho nesta classe $(100 \%)$, o que justifica a sua importância. Por fim, a terceira foi "paradoxo: mau e bom", por apresentar palavras que tanto remetem aos riscos como aos benefícios dos anabolizantes, pois tanto geram problemas renais $(100 \%)$ e impotência $(100 \%)$ como proporcionam agilidade $(100 \%)$ e resultado instantâneo $(100 \%)$, respectivamente.

$\mathrm{Na}$ definição "algo que aumenta a massa muscular", pode-se observar que a combinação entre anabolizantes e aumento da massa muscular está atrelada principalmente à ideia do imediatismo, e é motivada por questões estéticas, como se percebe a seguir: "Um adicional para quem quer ter um corpo perfeito mais rápido e um aumento repentino de massa muscular" [Participante (P) 003] e "É tipo uma droga injetável ou ingerida que provoca efeitos imediatos, como rápido crescimento muscular..." (P007). $\mathrm{O}$ resultado encontrado condiz com a literatura, visto que outros autores também extraíram um discurso "estético" dos sujeitos de suas pesquisas (Martins et al., 2005; Pedroso, 2012; L. S. M. F. Silva \& Moreau, 2003; S. H. A. Silva, 2013). Autores cujo público-alvo foi especificamente o adolescente, como Araújo (2003) e Wichstrøm e Pedersen (2001), observaram que os principais motivadores para a utilização dos esteroides pelos adolescentes são "melhora da performance no esporte" ou "melhora da aparência", e "para ficar bem" ou "para ser grande", respectivamente. Resultados que remetem a motivações, em sua maioria, estéticas.

No senso comum, principalmente entre os jovens, percebe-se que normalmente os anaboli- 
zantes são vinculados aos padrões de perfeição, até porque, o seu efeito anabólico corresponde à propriedade da testosterona em proporcionar um aumento da massa muscular (Iriart et al., 2009). Essa significação pode estar relacionada aos meios de comunicação e publicidade, os quais propagam um discurso de exaltação do corpo, dando a impressão de que ele pode ser transformado na hora e da forma que o sujeito quiser, sendo os anabolizantes, uma das alternativas para se alcançar tais objetivos (Iriart \& Andrade, 2002; Russo, 2005). Toda essa atratividade dos esteroides parece chamar atenção do público adolescente, já que eles relacionam os efeitos imediatos a consequências positivas e/ou uma espécie de recompensa, o que acaba tornando a rapidez dos efeitos anabólicos realmente cobiçada por quem almeja elevar sua autoestima e alcançar maior popularidade entre os pares. Logo, a massa muscular surge como um resultado desejável, todavia, o meio de se alcançar esta meta, caso seja por meio do uso dos anabolizantes, caracteriza-se como um perigoso atrativo.

Outro fator reforçador para o uso e que se relaciona com a concepção dos adolescentes sobre os anabolizantes, presente à pesquisa, refere-se ao senso de urgência da adolescência, isto é, da pressa como característica típica dessa fase, proveniente das repentinas mudanças físicas, sociais e psicológicas experimentadas por eles e, que acabam resvalando em suas escolhas e tendo implicações significativas na saúde do adolescente (Oliveira, 2012; Ribeiro, 2001). Assim, o uso de anabolizantes pode significar uma tentativa de "burlar" a natureza, acelerando o processo quando não se está satisfeito com o seu porte físico e se está em busca de um "corpo ideal".

A massificação desse discurso do "corpo ideal", conforme Oliveira (2012), tem sido absorvido especialmente pelos adolescentes e pode estar contribuindo significativamente para a definição que eles tem dos esteroides e para o aumento do uso por esse público, com o intuito de desenvolver a massa muscular rapidamente. Em seu estudo, os adolescentes apresentaram alto nível de satisfação de corporeidade quando passaram por mudanças corporais decorrentes dos anabolizantes, evidenciadas, especificamen- te, pelo aumento dos músculos. No presente estudo, também foi encontrada a necessidade de satisfação corporal, a exemplo da fala: "É uma substância química que é aplicada em pessoas que não estão satisfeitas com o seu próprio corpo para obter mais volume..." (P262).

Devido ao destaque dos efeitos benéficos dos anabolizantes, percebe-se que não se coloca em primeiro plano, necessariamente, os riscos, pois a atenção e o objetivo do uso concentram-se, primariamente, no aumento de massa muscular, ou seja, trata-se de uma busca desenfreada pela satisfação em detrimento aos prejuízos. Essa concepção também é encontrada em estudos com atletas (Aquino, 2001; Estevão \& Bagrichevsky, 2004), inclusive em atletas adolescentes (Araújo, 2003; Pérez, 2001). No estudo de Estevão e Bagrichevsky (2004), na opinião das fisiculturistas entrevistadas, ficar sem os esteroides é que se constitui em riscos, os quais podem ser o de não atingir a massa muscular e força planejada, e consequentemente perder as competições. No Brasil, a preocupação maior é com o jovem adolescente e não apenas com os fisiculturistas, pois, em seu imediatismo, quer ganhar massa e músculos rapidamente (Ribeiro, 2001; Souza \& Fisberg, 2002).

A segunda definição dos adolescentes para os anabolizantes, que foi "melhora o desempenho na academia", apontou a associação entre os esteroides e musculação: "É uma substância muito usada para aumentar o desempenho do corpo mais rápido na musculação..." (P017); e "É um tipo de droga que se usa para ter melhor resultado na academia..." (P397). A conceituação apontada confirma o argumento de A. G. Machado e Ribeiro (2004), a qual expressa que os esteroides tem se constituído num novo mito, por serem vistos como substâncias disponíveis para ganho de massa muscular e consequente melhoria do rendimento e do desempenho físico.

O somatório anabolizantes e musculação parece ter uma "justificativa" por parte dos adolescentes: "É um tipo de hormônio que os praticantes de musculação tomam quando não estão satisfeitos com o desenvolvimento muscular" (P127). A literatura confirma o exposto, ao mostrar que muitos usuários de anabolizantes 
optam pela sua utilização, por não conseguirem o aumento esperado de massa muscular somente com a musculação ou na velocidade que imaginam (Estevão \& Bagrichevsky, 2004; Wright, Grogan, \& Hunter, 2000). Daí volta-se novamente ao problema do imediatismo, visto que os efeitos visíveis e relativamente duradouros dos anabolizantes costumam atrair aqueles que têm pressa para obter resultados considerados satisfatórios.

Outra informação encontrada é que a busca pelo "corpo perfeito" entre os adolescentes parece estar associada à "fórmula" prática da musculação e uso de esteroides, com o intuito de provocar alterações corporais e com a concepção de que um depende do outro para a obtenção do resultado esperado, como expressam os respondentes: "Ficar marombado, diferente de quem pratica somente exercício fisico..." (P191) e "Melhor definição dos músculos, mais disposição para fazer atividade física e dá melhor resultado na academia" (P382). Autores como Firmino, Silva e Santos (2011) e Iriart et al. (2009) encontraram que as principais alterações corporais almejadas com o uso de anabolizantes são o peso, o crescimento muscular e definições corporais, visualizadas por um corpo sarado, definido e sem gordura.

Todo esse "culto ao corpo" tem sido impulsionado pelas academias, locais favoráveis para a predisposição ao uso dos esteroides e verdadeiros "templos da musculação" (Santos et al., 2006), cujo público adolescente tem sido um dos principais frequentadores (L. S. M. F. Silva \& Moreau, 2003), e ambiente este que tem incitado o drive for muscularity, ou seja, a preocupação exagerada com o corpo (Campana et al., 2014). A pesquisa de Campana et al., por exemplo, tanto indicou associações entre as variáveis de drive for muscularity e os hábitos de prática de atividade física, quanto que o uso de anabolizantes está dentre os principais chamados comportamentos negativos do citado construto. $\mathrm{O}$ mencionado investimento na musculatura também pode ser identificado no presente trabalho, revelando-se através de um efeito exagerado, mas que não parece ser visto de forma negativa por quem deseja ser cada vez mais admirado e aceito pelos pares:
"Produto que afeta especificamente o corpo da pessoa, tornando os seus músculos totalmente bombados, exagerados e duros" (P277); e "Uma técnica na qual as pessoas tomam para conseguir rapidamente um bom corpo ou um corpo exagerado..." (P429).

A prática de musculação na academia sem orientação profissional também facilita a predisposição aos anabolizantes, uma vez que contribui para a exposição a eles, por não conhecerem os efeitos reais do uso dessas substâncias (Oliveira, 2012; Wichstrøm \& Pedersen, 2001). Através dos discursos, percebe-se que os adolescentes costumam associar o uso, mesmo estético, a uma orientação profissional, como também que muitos deles exaltam o uso dos esteroides e desprezam a academia, com a crença de que as substâncias suprirão os exercícios físicos: “ $E$ uma droga injetável que as pessoas usam sem que precise ir a uma academia, por isso tomam anabolizantes demais e sem prescrição médica" (P052); e ". . . a pessoa acaba ficando com os músculos bem definidos, mas sempre tem que ter consulta médica" (P467). Conforme Oliveira (2012), estudos já tem apontado a desvinculação entre anabolizantes e musculação por parte dos respondentes, mas ele também ressalta que essa informação ainda precisa ser mais bem investigada.

Sabe-se que a adolescência se constitui em uma fase de desenvolvimento, cuja opinião dos pares é diferenciadamente considerada e, no que diz respeito ao tema anabolizantes, investigações já indicam que os iguais também exercem significativa influência para tal uso, uma vez que, nos casos de aplicação de anabolizantes com adolescentes, foram colegas ou amigos que ofereceram bem como aplicaram (Araújo, 2003; Wichstrøm $\&$ Pedersen, 2001). Tal resultado parece indicar que estes sejam os maiores influenciadores para o contato e início do uso de anabolizantes.

Manetta e Silveira (2000) destacam que o instrutor de educação física seria um segundo influenciador, por normalmente ser quem apresenta, receita e vende os anabolizantes, mesmo sem nenhum conhecimento na área, além das próprias academias, que comercializam os produtos sem qualquer critério e controle, o que demonstra a 
facilidade para a obtenção das substâncias. Desse modo, percebe-se que a mídia, as academias, os amigos e instrutores parecem exercer uma influência social significativa para o adolescente, principalmente os amigos, mediante a necessidade de aprovação e aceitação no grupo. De acordo com Oliveira (2012), ter o corpo diferente dos amigos costuma gerar sofrimento e marginalização, o que pode fazer dos esteroides uma espécie de "atalho" para ser inserido nestes contextos que valorizam a beleza e o corpo musculoso.

Por sua vez, a última definição desta Classe, o "paradoxo: mau e bom", mesmo sendo a terceira conceituação, indicou que os principais termos ("rim", "impotência", "agilidade", e instantâneo") possuem a frequência total na Classe e um sentido contraditório. O paradoxo é constatado porque, segundo os respondentes, ao mesmo tempo em que os anabolizantes trazem benefícios, eles também acarretam riscos/malefícios aos seus usuários, tais como: "A pessoa fica musculosa, ganha mais agilidade, disposição e resistência, mas é prejudicial..." (P322); "Um produto que aumenta a massa muscular rapidamente, deixa forte, mas causa impotência sexual..." (P323); e "Substância que estimula o crescimento instantâneo dos músculos, mas agride os rins..." (P381).

Sobre os efeitos colaterais, a literatura informa que o câncer e a impotência sexual são os mais conhecidos, explicando que a possível causa se deve à mídia, por se utilizar do sensacionalismo e divulgar as consequências consideradas mais graves (Ribeiro, 2001). O câncer não foi o mais mencionado neste estudo, mas também teve alta frequência $(60 \%)$, já a impotência sexual (100\%) parece ser um problema temido pelos adolescentes, provavelmente porque o órgão sexual masculino é considerado o maior representante da masculinidade.

Essa noção de anabolizantes como algo bom, porém com ressalvas, também foi detectada noutros estudos (Iriart \& Andrade, 2002; Iriart et al., 2009; Santos et al., 2006). Este último autor, por exemplo, encontrou que dentre os usuários de anabolizantes, mesmo os que deixaram o uso, a maioria se mostrou satisfeita com os resultados obtidos com os esteroides. Este achado é representativo, pois leva à conclusão de que, apesar do risco, parece ter valido a pena, o que retoma o problema, uma vez que significa subjugar os prejuízos cumulativos do uso indiscriminado da substância. Essa questão pode ser ainda mais problemática entre os adolescentes, já que nesta fase a busca pelo estabelecimento da própria identidade é mais evidente, podendo colaborar para a prática de comportamentos negativos ou inadequados, como o uso de anabolizantes (Oliveira, 2012).

Observa-se que esse dilema entre o risco e o desejo é comum entre os usuários de drogas ilícitas, pois, ao mesmo tempo em que eles reconhecem os malefícios do uso, também confessam que elas trazem "benefícios", como a sensação de liberdade, prazer e esquecimento dos problemas (Braz, 2008). Comparado a outras drogas de abuso, os anabolizantes não são fortemente euforizantes (Amsterdam et al., 2010) e não são utilizados para alcançar um efeito imediato de intoxicação aguda, mas normalmente são consumidos em ciclos, para a obtenção de uma recompensa posterior de aumento da musculatura (Kanayama, Brower, Wood, Hudson, \& Pope, 2009). Mesmo assim, tanto os efeitos de bem-estar como os disfóricos de retirada podem contribuir para um quadro de síndrome de dependência (Amsterdam et al., 2010; Kanayama et al., 2009).

Nesse paradoxo entre o desejo de crescer e ficar forte e o perigo quanto ao uso de anabolizantes, eis a questão: quando e como se decide sobre qual pesa mais? No estudo de Oliveira (2012), mesmo afirmando não saber o que são, os adolescentes declaram assumir o risco de utilização com objetivo estético. Já no de Estevão e Bagrichevsky, (2004), apesar dos usuários conhecerem os esteroides e seus efeitos colaterais, insistem em utilizá-los para uma suposta melhoria de seu corpo. Os resultados mencionados parecem indicar que a variável "conhecimento da substância" não interfere no poder de decisão, mas sim o fato dos anabolizantes não mostrarem, em geral, consequências negativas imediatas. Sendo assim, passa-se uma imagem de satisfação e saúde, formada pelo discurso de uma falsa "aparência saudável”, visualizada pelos mús- 
culos e corpo torneados, fazendo da aparência uma mediadora do risco para a saúde do indivíduo (Santos et al., 2006), e desprezando-se os efeitos a longo prazo (Russo, 2005). Sobre esta noção de satisfação, também foi encontrada uma ambiguidade entre saúde e doença no presente trabalho, pois enquanto alguns associam o uso de anabolizantes a um corpo saudável, outros relacionam à perda da saúde: "Algum remédio que ajuda o metabolismo, ou melhor, acelera com exercícios físicos e uma boa alimentação a ter um corpo saudável" (P103); e "Para quem tinha uma vida saudável passa a ter uma vida com a saúde fraca" (P435).

Os adolescentes também incluíram a orientação médica como um suposto fator positivo para quem utiliza os esteroides, fato que é verdadeiro para aqueles que fazem uso mediante necessidade clínica. Todavia, a forma como o sujeito a seguir se expressa, parece indicar um desvio de função da substância para fins estéticos: "Se for tomado ou aplicado com orientação médica será benéfico, saudável e vai ter músculos rápido" (P133).

Estudos mostram que usuários possuem a crença de que os efeitos adversos podem ser controlados e/ou evitados com o uso de outros medicamentos e/ou acompanhamento médico (L. S. M. F. Silva \& Moreau, 2003). Portanto, alguns adolescentes demonstram um conhecimento equivocado acerca dos anabolizantes e, mesmo aqueles que possuem certo conhecimento parecem estar suscetíveis à atração pelos anabolizantes, o que reforça ainda mais a existência de uma concepção baseada num conflito entre "o querer e o certo a fazer" e demonstra que eles tem alguma noção do perigo, mas os ganhos parecem ser maiores ou o risco parece ser aceitável.

\section{Classe 2: Benefícios Derivados dos Anabolizantes (19,1\%)}

A Classe 2 é formada pelos benefícios que os anabolizantes geram, de acordo com a opinião dos adolescentes em estudo. A partir da sua análise, percebeu-se que se trata de uma Classe consensual, visto que, apesar de agrupar o menor número de segmentos $(132=19,1 \%)$, a maioria dos termos remete à imagem corporal. Por este motivo, a partir de um discurso simbólico, a Classe foi denominada: "Ao tomar anabolizantes, a pessoa fica com uma boa aparência, autoestima elevada e chama atenção". As falas a seguir expressam a nomenclatura da Classe: "A satisfação de olhar para o espelho e se sentir bonito, forte e sarado, ou seja, o aumento da autoestima com a boa aparência" (P259); e "A aparência da pessoa muda e o corpo fica maior, mais esbelto, atrativo e chama atenção, tudo isso em pouco tempo" (P372). Conforme Russo (2005), na contemporaneidade, olhares direcionam-se para o corpo e, através dos meios de comunicação, criam-se desejos e padronizam corpos. Aqueles que não conseguem se moldar ao padrão, sentem-se cobrados e insatisfeitos, e muitos iniciam uma corrida em busca da aparência física idealizada.

Autores como A. G. Machado e Ribeiro (2004) e Ribeiro (2001) destacam que, na linguagem dos jovens, "bombar" significa ficar mais atraente para as garotas e mais forte e poderoso entre os pares, ou seja, popular. Como o uso inicial de anabolizantes gera um resultado que impressiona o adolescente, este normalmente é influenciado pelos elogios (Ribeiro, 2001). Os achados desta pesquisa parecem estar em harmonia com os dos citados autores porque a popularidade, segundo os adolescentes, pode ser evidenciada pelos olhares e pelos cobiçados elogios: "Melhora a autoestima porque a pessoa fica forte com facilidade, chama atenção por estar forte e fica mais feliz quando as pessoas elogiam pelo crescimento" (P220); e "Aumentam a autoestima, se sentem muito mais bonitas e vaidosas, principalmente quando recebem um elogio" (P375).

Partindo do fato de que a adolescência é a fase em que as primeiras paixões ou romances são despertados, o apelo romântico também parece estar associado ao uso de anabolizantes para os adolescentes dessa pesquisa: "Elevação da autoestima, fica bombado e chama atenção das pessoas, principalmente das mulheres..." (P312). Tanto Araújo (2003) quanto Wichstrøm e Pedersen (2001), defendem essa hipótese porque os jovens de suas pesquisas declararam que uma das razões principais para a boa aparência 
por meio do uso de anabolizantes, é ter maiores chances com o sexo oposto.

Observou-se também que, em praticamente todos os discursos citados, os respondentes mencionaram o fator autoestima, mais especificamente a necessidade de sua elevação, colocando-a como um construto relevante para a imagem corporal do adolescente, e citando o anabolizante como uma suposta solução para tal problema, fato constatado principalmente nas falas a seguir: “. . . são bons para o psicológico de algumas pessoas..." (P162); e "Muitos usam para ter uma boa aparência ou porque gostam de se aparecer, no entanto eles usam para tentar aumentar a sua autoestima" (P354). Tais opiniões expressam carência em se sentir bem e, conforme autores da área, a baixa autoestima seria uma característica de personalidade comum em usuários de anabolizantes, configurando o seu uso como uma estratégia utilizada para compensar esses sentimentos de baixa autoestima ou outros problemas emocionais mais graves, como o transtorno dismórfico corporal ou vigorexia, uma vez que, nestes casos, mesmo com um ganho muscular enorme, o sujeito sempre se vê fraco em comparação a outrem (Araújo, 2003; Manetta \& Silveira, 2000; Oliveira, 2012; Russo, 2005).

Portanto, é fato que vários elementos podem contribuir para uma concepção positiva e consequente utilização dos anabolizantes na adolescência, tais como a insatisfação com a aparência física, representada pela baixa autoestima; necessidade de chamar atenção, ou seja, de ser popular; de aprovação e/ou aceitação do grupo; e até a possibilidade de se tornar um símbolo sexual, configurando-se então, em fatores que exercem um grande impacto na vida do adolescente (Araújo, 2003; A. G. Machado \& Ribeiro, 2004; Ribeiro, 2001). Além destes, a venda ilegal também contribui significativamente para o fácil acesso aos esteroides.

\section{Classe 3: Prejuízos Derivados dos Anabolizantes (41,6\%)}

Esta última classe refere-se aos malefícios causados pelos anabolizantes, conforme a percepção dos respondentes. Ela apresentou maior predominância de segmentos $(288=41,6 \%)$, mas também o menor número de palavras exclusivas na Classe $(p<0,0001=12$ palavras $)$. Tal diversidade permitiu nomeá-la da seguinte maneira: "Não trazem nenhum beneficio, pois podem causar graves consequências, como doenças, amputação de membros e até a morte".

A conclusão de que os adolescentes da pesquisa não veem benefícios com o uso de anabolizantes foi constatada pelos termos mais importantes desta categoria (nenhum benefício: $X^{2}=148,91$ ), além das respostas: "Para mim anabolizantes tem beneficios em animais, se tiver algum beneficio para os seres humanos eu desconheço" (P013); e "Nenhum benefício, pois é algo que só pessoas com mente fraca usam..." (P339). Sobre a opinião negativa acerca dos esteroides, autores como Wright et al. (2000) defendem que os não usuários estão mais sujeitos a descrever os efeitos colaterais físicos negativos dessas substâncias, visto que, se não as utilizam, provavelmente é porque possuem uma opinião depreciativa a respeito. Apesar da presente pesquisa não ter entrado neste mérito de uso, percebeu-se que a forma como os adolescentes se expressaram pareceu ser a de um não usuário, por se distanciarem do objeto: " $E$ uma droga que algumas pessoas sentem prazer em usar, mas eu não vejo beneficio nenhum em relação ao uso" (P079); e "Só quem usa sabe realmente como responder, mas não vejo nenhum beneficio nisso..." (P136).

Vale destacar ainda que, o uso animal, apontado pelo sujeito 013 , também foi mencionado por vários outros respondentes, inclusive fazendo menção ao cavalo, animal mais associado aos anabolizantes. Sabe-se que, embora haja esteroides de uso exclusivo veterinário (Ferrão \& Bressan, 2006), também há aqueles autorizados para o uso humano, desde que, com a finalidade clínica, como para o tratamento de doenças como o câncer, osteoporose e até patologias descobertas na puberdade e relacionadas ao desenvolvimento (Amsterdam et al., 2010; Martins et al., 2005; F. L. Rocha et al., 2007). Outro fato encontrado por parte dos sujeitos da pesquisa e que chamou atenção, diz respeito à frequência da resposta "não sei" (85,7\%): "Não sei muito bem sobre os anabolizantes, mas pelo que eu sei fazem mal ao ser humano...” (P091); e “. . . no início o resul- 
tado é bom, mas depois aparecem as consequências, doenças, só não sei quais" (P253). Os mencionados argumentos parecem indicar um fraco conhecimento a respeito, o que pode representar riscos e levar a uma subestimação dos efeitos colaterais decorrentes do uso (Araújo, 2003).

A análise da classe também apontou que ela possui o maior número de palavras significantes com frequência máxima (100\%), a exemplo de sequelas, inchaço, mata, depois, lindo, e adolescente. Dentre elas, os termos depois e lindo, apesar de parecerem desconectados à primeira vista, referem-se à opinião de que o uso de anabolizantes parece ser benéfico inicialmente e até "lindo", mas depois vem as consequências: "No começo fica com o corpo maravilhoso, lindo e sarado depois fica um lixo todo mole" (P292); "Boa forma no início, mas depois várias consequências negativas..." (P158). A palavra adolescente também se destacou, visto que as falas relacionadas mostram que o próprio sujeito se reconhece fazendo parte de um grupo vulnerável: "É uma droga na qual as pessoas usam, geralmente adolescentes, e em alguns casos traz os músculos tão sonhados, mas pode trazer consequências terriveis" (P128); e "É um tipo de comprimido que geralmente é utilizado pelos adolescentes para ficar forte, mas pode deixar sequelas ou levar à morte" (P236).

Quanto às consequências graves levantadas pelos adolescentes, as mais relevantes e que resumem todos os problemas citados são morte, doenças e amputações, por ordem de evocação. A primeira seria o extremo do malefício dos anabolizantes, o que a literatura expõe não ser frequente, mas que pode ocorrer em casos mais severos e associados a outros fatores de vulnerabilidade (S. H. A. Silva, 2013). Em seguida são as doenças, as quais foram definidas pelos respondentes como problemas e citadas de forma bastante variada, porém, a depender da gravidade, podem ser revertidas: problemas cardíacos, pulmonares, hepáticos, sanguíneos, de pele, psicológicos, renais, impotência, câncer, etc. Dentre eles, destacaram-se os problemas de pele, sendo a presença de acnes um efeito colateral comum (Amsterdam et al., 2010), o que para o adolescente que prioriza a beleza não parece ser nada agradável; e os problemas psicológicos, dentre eles a agressividade, uma alteração comportamental que pode trazer sérios danos para o indivíduo e para o meio social em que ele vive (Martins et al., 2005). Por fim, a amputação de membros, que representa um dano drástico, irreversível, e infelizmente frequente, devido à administração inconsequente e altamente abusiva dos anabolizantes (Brandi \& Carneiro, 2010).

Outro termo que chamou atenção foi saúde, em virtude dos adolescentes relacionarem a palavra a consequências negativas, riscos. Todavia, esses danos são remetidos ao futuro, palavra que foi constantemente relacionada: ". . . podem acarretar em algumas consequências que prejudicarão a saúde no futuro" (P111); e "são prejudiciais à saúde, pois geram graves consequências e danos futuros..." (P372).

Por fim, a reprovação familiar e/ou por pessoas não coniventes com o uso de anabolizantes foram situações apontadas por alguns respondentes: “. . . pode se sentir rejeitada por olhares das demais pessoas que reprimem essa prática" (P73); e ". . a as pessoas se sentem constrangidas na frente de sua familia" (P351). Apesar de pessoas próximas, como familiares e até amigos, desaprovarem o uso de anabolizantes, observa-se que essa reprovação não tem sido suficiente para impedir que muitos adolescentes adotem essa prática, uma vez que as pressões socioculturais com o seu padrão de beleza estabelecido parecem ser condições determinantes para o desenvolvimento de distorções da imagem corporal, em especial em adolescentes (Oliveira, 2012).

Portanto, apesar dos adolescentes terem reconhecido que os anabolizantes não trazem nenhum benefício, além de citarem inúmeros prejuízos provocados por eles, muitos possuem uma concepção deficiente a respeito. Em outras palavras, os respondentes sabem que os anabolizantes causam danos, mas não conhecem especificamente o grau de alcance dos seus efeitos colaterais, assim como expressam ambivalência na avaliação de seus efeitos.

\section{Considerações Finais}

A presente pesquisa objetivou conhecer o conceito de anabolizantes, bem como os benefícios e malefícios do uso, conforme a opinião 
dos adolescentes. Na classe 1 verificou-se que as duas primeiras definições apresentam aspectos positivos para os adolescentes, todavia a terceira expressa uma visão mais ambígua, visto que eles descrevem muitos benefícios sociais, e só no final do discurso é que informam os danos, dando a impressão de que os ganhos parecem ser maiores ou o risco parece ser aceitável.

A Classe 2, relacionada aos benefícios propriamente ditos, demonstrou que os adolescentes não destacam apenas os ganhos, mas também os custos, apesar destes nem sempre serem considerados e das vantagens serem muito mais ressaltadas. Por fim, a Classe 3 indicou que os adolescentes possuem certo conhecimento dos malefícios dos anabolizantes, mas que essa concepção, se superficial, pode não ser suficiente para impedir que o adolescente se sinta atraído pelos resultados dos esteroides a curto prazo.

As três classes expressam, por meio do discurso dos adolescentes, que eles reconhecem a preocupação de uma notável parcela desta população em alcançar a aparência física idealizada. Também foi comum a todas as classes um discurso voltado para a saúde, apenas diferenciando a maneira de perceber esse conceito, uma vez que, enquanto na primeira classe a saúde está implícita no discurso de uma falsa "aparência saudável”, nas duas últimas os adolescentes correlacionam a saúde a consequências negativas.

Em meio à adolescência, fase de desenvolvimento em que o público-alvo se encontra, percebem-se características típicas desse período que podem colaborar para concepções errôneas ou equivocadas, como o senso de urgência e a dificuldade em estabelecer a própria identidade, e também fatores internos e externos, como a baixa autoestima, popularidade, reconhecimento social, venda ilegal de anabolizantes, contato com o mundo das academias, a pressão social, o culto ao corpo, dentre outros, os quais podem abrir uma janela de vulnerabilidade aos anabolizantes.

A presente pesquisa não entrou no mérito de uso ou não dos anabolizantes por parte do público em questão, sendo uma sugestão para os próximos trabalhos, visto que se abre a possibilidade de extrair discursos mais específicos daqueles que nunca usaram, dos que usam e dos que porventura tenham deixado de utilizar os esteroides, a fim de identificar a concepção predominante em cada grupo. Um aspecto importante a se destacar é que o estudo não se baseou em uma amostra representativa da população de estudantes do ensino médio em Sergipe, limitando sua capacidade de generalização dos achados. É também uma limitação o fato de ter sido feito com uma amostra exclusivamente extraída de escolas públicas, já que a comparação entre pública e privada poderia revelar diferentes percepções sobre o tema, o que fica como indicação de pesquisa para futuras investigações.

Mesmo com as citadas limitações, é possível detectar que se faz necessário o desenvolvimento de uma política que expanda a divulgação de informações sobre o uso e das consequências dessas substâncias, visto que o conhecimento demonstrado pelos adolescentes se mostrou ambíguo. A literatura também compartilha o conflito entre ganhos e perdas, vivenciado pelos adolescentes, o que demonstra que nem todos estão desinformados e que medidas preventivas não devem ser baseadas apenas na ênfase aos malefícios, mas no balanceamento dos argumentos, a fim de que os adolescentes optem por decisões sensatas. A exemplo disso, podem ser implementadas em locais estratégicos, tais como escolas ou academias, ações educativas que abordem como a autoestima e a aparência podem influenciar a favorabilidade aos anabolizantes.

\section{Referências}

Alan, I. (2009). Research report series. National Institute on Drug Abuse. Retrieved from https:// www.drugabuse.gov/publications/finder/t/162/ ResearchReports

Almeida, I. S., Rodrigues, B. M. R. D., \& Simões, S. M. F. (2007). O adolescer... Um vir a ser. Adolescência \& Saúde, 4(3), 24-28.

Amsterdam, J. V., Opperhuizen, A., \& Hartgens, F. (2010). Adverse health effects of anabolic-androgenic steroids. Regulatory Toxicology and Pharmacology, 57, 117-123. doi:10.1016/j. yrtph.2010.02.001

Antunes, C., Sousa, M. C., Carvalho, A., Costa, M., Raimundo, F., Lemos, E., ...Andrade, A. (2006). Auto-estima e comportamentos de saú- 
de e de risco no adolescente: Efeitos diferenciais em alunos do $7^{\circ}$ ao $10^{\circ}$ ano. Psicologia, Saúde \& Doenças, 7, 117-123.

Aquino, F. R., Neto. (2001). O papel do atleta na sociedade e o controle de dopagem no esporte. Revista Brasileira de Medicina do Esporte, 7(4), 138148. doi:10.1590/S1517-86922001000400005

Araújo, J. P. (2003). O uso de esteróides androgênicos anabolizantes entre estudantes do ensino médio do Distrito Federal (Dissertação de mestrado, Programa de Pós-Graduação em Educação Física, Universidade Católica de Brasília, DF, Brasil).

Brandi, C. R., \& Carneiro, M. A., Jr. (2010). Esteróides anabólicos androgênicos (EAAS): O que são e quais os seus efeitos sobre o organismo humano? EFDeportes.com, Revista Digital, 15(148). Recuperado em www.efdeportes.com/efd148/esteroides-anabolicos-androgenicos-oque-sao.htm

Braz, R. A. (2008). O combate às drogas através da educação. Maringá, PR: Universidade Estadual de Maringá. Recuperado em http://central3. to.gov.br/arquivo/195016/

Camargo, B. V. (2005). ALCESTE: Um programa informático de análise quantitativa de dados textuais. In A. S. P. Moreira, B. V. Camargo, J. C. Jesuíno, \& S. M. Nóbrega (Eds.), Perspectivas teórico-metodológicas em representações sociais (pp. 511-539). João Pessoa, PB: Editora da Universidade Federal da Paraíba.

Camargo, B. V., \& Justo, A. M. (2013). IRAMUTEQ: Um software gratuito para análise de dados textuais. Temas em Psicologia, 21, 513-518. doi:10.9788/TP2013.2-16

Campana, A. N. N. B., Morgado, J. J. M., Morgado, F. F. R., Campana, M. B., Ferreira, L., \& Tavares, M. C. G. C. F. (2014). Drive for muscularity: Um estudo exploratório no exército brasileiro. Psicologia: Teoria e Pesquisa, 30, 213-222. doi:10.1590/s0102-37722014000200011

Centro Brasileiro de Informações sobre Drogas Psicotrópicas. (2010). VI Levantamento Domiciliar sobre o uso de drogas psicotrópicas entre estudantes do ensino fundamental e médio das redes públicas e privadas de ensino nas 27 capitais no Brasil - 2010. São Paulo, SP: Universidade Federal de São Paulo.

Darke, S., Torok, M., \& Duflou, J. (2014). Sudden or unnatural deaths involving anabolic-androgenic steroids. Journal of Forensic Sciences, 59(4), 1025-1028. doi:10.1111/1556-4029.12424
Estevão, A., \& Bagrichevsky, M. (2004). Cultura da corpolatria e body-building: Notas para reflexão. Revista Mackenzie de Educação Física e Esporte, 3(3), 13-25.

Ferrão, S. P. B., \& Bressan, M. C. (2006). O uso de agentes anabolizantes na produção de carnes e suas implicações - Revisão. Veterinária Notícias, 12, 69-78.

Firmino, L. Y. L., Silva, M. G., \& Santos, C. R. (2011). O uso de esteroides anabolizantes por praticantes de musculação. Trindade, GO: Faculdade União de Goyazes.

Iriart, J. A. B., \& Andrade, T. M. (2002). Musculação, uso de esteróides anabolizantes e percepção de risco entre jovens fisiculturistas de um bairro popular de Salvador, Bahia, Brasil. Cadernos de Saúde Pública, 18, 1379-1387. doi:10.1590/ S0102-311X2002000500031

Iriart, J. A. B., Chaves, J. C., \& Orleans, R. G. (2009). Culto ao corpo e uso de anabolizantes entre praticantes de musculação. Cadernos de Saúde Pública, 25, 773-782. doi:10.1590/S0102$-311 X 2009000400008$

Kanayama, G., Brower, K. J., Wood, R. I., Hudson, J. I., \& Pope, H. G., Jr. (2009). Issues for DSM-V: Clarifying the diagnostic criteria for anabolicandrogenic steroid dependence. American Journal Psychiatry, 166, 642-644. doi:10.1176/appi. ajp.2009.08111699

Machado A. G., \& Ribeiro P. C. P. (2004). Anabolizantes e seus riscos. Adolescência \& Saúde, (4), 20-22.

Machado, L. M. G. S. (2010). Crenças e Representações Sociais dos adolescentes sobre a violência interpessoal (Dissertação de mestrado, Universidade Fernando Pessoa, Porto, Portugal).

Manetta, M. C. P., \& Silveira, D. X. (2000). Uso abusivo de esteroides androgênicos anabolizantes. Psiquiatria na Prática Médica, 33(4). Recuperado em http://www.unifesp.br/dpsiq/polbr/ ppm/atu1_04.htm

Martins, C. M., Carijó, F. H., de Almeida, M. C., da Silveira, M., Mirailh, M. X. N., Peixoto, M. M., ...Sholl-Franco, A. (2005). Efeitos psicológicos do abuso de anabolizantes. Ciências \& Cognição, 5, 84-91.

Nangia, A. K. (2014). Anabolic steroid abuse: A paradox of manliness. Fertility and Sterility, 101(5), 1247. doi:10.1016/j.fertnstert.2014.02.034 
Oliveira, U. (2012). O uso de esteroides androgênicos anabolizantes entre adolescentes e sua relação com a prática da musculação (Tese de doutorado, Universidade de Campinas, SP, Brasil). Recuperado em http://www.unicamp.br/unicamp/sites/default/files/jornal/paginas/ju_559_ paginacor_09_web.pdf

Ozella, S. (2002). Adolescência: Uma perspectiva crítica. In M. L. J. Contini, S. H. Koller, \& M. N. S. Barros (Eds.), Adolescência \& Psicologia: Concepções, práticas e reflexões críticas (pp. 16-24). Rio de Janeiro, RJ: Conselho Federal de Psicologia.

Pagonis, T. A., Angelopoulos, N. V., Koukoulis, G. N., \& Hadjichristodoulou, C. S. (2005). Psychiatric side effects induced by suprafisiological doses of combinations of anabolics steroids correlate to the severity of abuse. European Psychiatry, 21(8), 551-562. doi:10.1016/j.eurpsy.2005.09.001

Pedroso, J. L. (2012). Esteróides anabolizantes e o sistema nervoso. Revista Neurociência, 20(2), 181-182.

Pérez, M. B. (2001). Uso de anabólicos por atletas adolescentes. Revista de Endocrinología y $\mathrm{Nu}$ trición, 9(3), 133-140.

Pope, H. G., Jr., \& Kanayama, G. (2012). Anabolicandrogenic steroids. In J. C. Vester, K. Brady, M. Galanter, \& P. Conrod (Eds.), Drug abuse and addiction in medical illness: Causes, consequences and treatment (pp. 251-264). Boston, MA: Springer. doi:10.1007/978-1-4614-33750_19

Ribeiro, P. C. P. (2001). O uso indevido de substâncias: Esteróides anabolizantes e energéticos. Adolescência Latinoamericana, 2(2), 97-101.

Rocha, F. L., Roque, F. R., \& Oliveira, E. M. (2007). Esteróides anabolizantes: Mecanismos de ação e efeitos sobre o sistema cardiovascular. O Mundo da Saúde, 31(4), 470-477.

Rocha, M., Mota, C. P., \& Matos, P. M. (2011). Vinculação à mãe e ligação aos pares na adolescência: O papel mediador da autoestima. Análise Psicológica, 2(29), 185-200. doi:10.14417/ap.47

Russo, R. C. T. (2005). Imagem corporal: Construção através da cultura do belo. Movimento e Percepção, 5(6). Recuperado em www.unipinhal.edu.br

Santos, A. F., Mendonça, P. M. H., Santos, L. A., Silva, N. F., \& Tavares, J. K. L. (2006). Ana- bolizantes: Conceitos segundo praticantes de musculação em Aracaju (SE). Psicologia em Estudo, 11, 371-380. doi:10.1590/S141373722006000200016

Silva, L. S. M. F., \& Moreau, R. L. M. (2003). Uso de esteróides anabólicos androgênicos por praticantes de musculação de grandes academias da cidade de São Paulo. Revista Brasileira de Ciências Farmacêuticas, 39, 327-333. doi:10.1590/ S1516-93322003000300012

Silva, S. H. A., Jr. (2013). Morbidade hospitalar por ingestão de esteroides anabólico-androgênicos (EAA) no Brasil. Revista Brasileira de Medicina do Esporte, 19, 108-111. doi:10.1590/S151786922013000200007

Simões, C. (2010). Adolescentes e comportamentos de saúde. Alicerces, 3(3), 223-241.

Socas, L., Zumbado, M., Pérez-Luzardo, O., Ramos, A., Pérez, C., Hernández, J. R., \& Boada, L. D. (2005). Hepatocellular adenomas associated with anabolic androgenic steroid abuse in bodybuilders: A report of two cases and a review of the literature. British Journal of Sports Medicine, 39(5), 1-4. doi:10.1136/bjsm.2004.013599

Sociedade Brasileira de Endocrinologia e Metabologia. (2014). Anabolizantes esteroides e os jovens. Recuperado em http://www.endocrino.org. br/anabolizantes-esteroides-e-os-jovens/

Souza, E. S., \& Fisberg, M. (2002). O uso de esteróides anabolizantes na adolescência. Brazilian Pediatric News, 4, 31-34. Recuperado em http:// brazilpednews.org.br

Thiblin, I., \& Pärlklo, T. (2002). Anabolic androgenic steroids and violence. Acta Psychiatrica Scandinavica, 412, 125-128. doi:10.1034/j.16000447.106.s412.27.x

Wichstrøm, L., \& Pedersen, W. (2001). Use of anabolic-androgenic steroids in adolescence: Winning, looking good or being bad? Journal of Studies in Drugs and Alcohol, 62, 5-13. doi:10.15288/ jsa.2001.62.5

Wright, S., Grogan, S., \& Hunter, G. (2000). Motivations for anabolic steroid use among bodybuilders. Journal of Health Psychology, 5(4), 566-571. doi:10.1177/135910530000500413

Recebido: $10 / 03 / 2015$

$1^{a}$ revisão: $04 / 05 / 2015$ Aceite final: 08/05/2015 